\title{
Network Controllability in the Inferior Frontal Gyrus Relates to Controlled Language Variability and Susceptibility to TMS
}

\author{
John D. Medaglia, ${ }^{2,8}$ @Denise Y. Harvey, ${ }^{2,7}$ Nicole White, ${ }^{2}$ Apoorva Kelkar, ${ }^{2,8}$ ㄱared Zimmerman, ${ }^{3}$ \\ -Danielle S. Bassett, ${ }^{2,4,5,6}$ and Roy H. Hamilton ${ }^{2}$ \\ ${ }^{1}$ Department of Psychology, ${ }^{2}$ Department of Neurology, ${ }^{3}$ Department of Neuroscience, ${ }^{4}$ Department of Electrical and Systems Engineering, ${ }^{5}$ Department of \\ Bioengineering, ${ }^{6}$ Department of Physics and Astronomy, University of Pennsylvania, Philadelphia, Pennsylvania, $19104,{ }^{7}$ Moss Rehabilitation Research Institute, \\ Elkins Park, Pennsylvania, 19027, and ${ }^{8}$ Department of Psychology, Drexel University, Philadelphia, Pennsylvania, 19104
}

In language production, humans are confronted with considerable word selection demands. Often, we must select a word from among similar, acceptable, and competing alternative words to construct a sentence that conveys an intended meaning. In recent years, the left inferior frontal gyrus (LIFG) has been identified as being critical to this ability. Despite a recent emphasis on network approaches to understanding language, how the LIFG interacts with the brain's complex networks to facilitate controlled language performance remains unknown. Here, we take a novel approach to understanding word selection as a network control process in the brain. Using an anatomical brain network derived from high-resolution diffusion spectrum imaging, we computed network controllability underlying the site of transcranial magnetic stimulation (TMS) in the LIFG between administrations of language tasks that vary in response (cognitive control) demands: open-response tasks (word generation) versus closed response tasks (number naming). We found that a statistic that quantifies the LIFG's theoretically predicted control of communication across modules in the human connectome explains TMSinduced changes in open-response language task performance only. Moreover, we found that a statistic that quantifies the LIFG's theoretically predicted control of difficult-to-reach states explains vulnerability to TMS in the closed-ended (but not open-ended) response task. These findings establish a link among network controllability, cognitive function, and TMS effects.

Key words: cognitive control; connectomics; diffusion imaging; neural networks; neuroimaging; TMS

Significance Statement

This work illustrates that network control statistics applied to anatomical connectivity data demonstrate relationships with cognitive variability during controlled language tasks and TMS effects.

\section{Introduction}

Effective verbal communication depends on the ability to retrieve and select the appropriate words that correspond to a speaker's

Received Jan. 11, 2017; revised March 3, 2018; accepted April 25, 2018.

Author contributions: D.Y.H. edited the paper; J.D.M., D.Y.H., and R.H.H. designed research; J.D.M. and A.K. performed research; D.S.B. contributed unpublished reagents/analytic tools; J.D.M., D.Y.H., N.W., A.K., and J.Z. analyzed data; J.D.M. wrote the paper.

This work was supported by the National Institutes of Health (Office of the Director Grant 1-DP5-0D-021352-01 to J.D.M.; National Institute of Mental Health Grant 2-R01-DC-009209-11 to D.S.B.; and National Institute of Child Health and Human Development Grant 1R01HD086888-01 to D.S.B.); the John D. and Catherine T. MacArthur Foundation (D.S.B.); the Alfred P. Sloan Foundation (D.S.B.); the Army Research Laboratory and the Army Research Office (Grants W911NF-10-2-0022 and W911NF-14-1-0679 to D.S.B.); the Office of Naval Research (D.S.B.); the National Science Foundation (Grants BCS-1441502, BCS-1430087, and CAREER PHY-1554488 to D.S.B.); and the Perelman School of Medicine at the University of Pennsylvania (Translational Neuroscience Initiative grant to J.D.M., D.S.B., and R.H.H.).

The authors declare no competing financial interests.

Correspondence should be addressed to John D. Medaglia, Department of Psychology, Drexel University, 322 Stratton Hall, Philadelphia, PA 19104. E-mail: johnmedaglia@gmail.com.

DOI:10.1523/JNEUROSCI.0092-17.2018

Copyright $\odot 2018$ the authors $\quad 0270-6474 / 18 / 386399-12 \$ 15.00 / 0$ intended meaning. Often, the opportunity to select among several appropriate words challenges the speaker. Prior evidence in cognitive neuroscience indicates that the left inferior frontal gyrus (LIFG) supports verbal selection (Botvinick et al., 2001, Moss et al., 2005, Nelson et al., 2009, Snyder et al., 2011, Thompson-Schill et al., 1997, 1999, Thompson-Schill and Botvinick, 2006, Tippett et al., 2004) and potentially a more domain-general role in selection in the context ofcompetingrepresentations (FedorenkoandThompsonSchill, 2014). The position of the LIFG in the brain's distributed anatomical networks is not unique to classically described language systems; rather, it is positioned to mediate between several systems in the frontal associative, motor, insular, and temporal cortices as well as the basal ganglia (Saur et al., 2008). This evidence suggests that the participation of the LIFG in language function must operate in the context of many processing demands including language production (Benedek et al., 2014, Cai et al., 2013, Gernsbacher and Kaschak, 2003, Segaert et al., 2012, Ye and Zhou, 2009) and cognitive control in the brain's distributed circuits. 
A

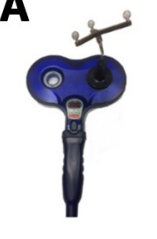

$50 \mathrm{~Hz} @ 5 \mathrm{~Hz}$

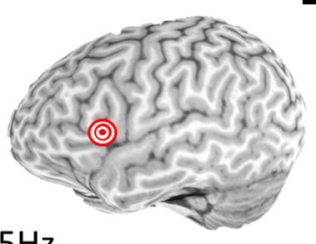

B

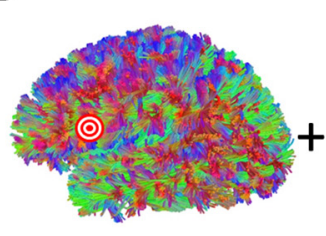

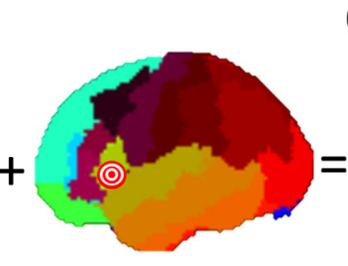

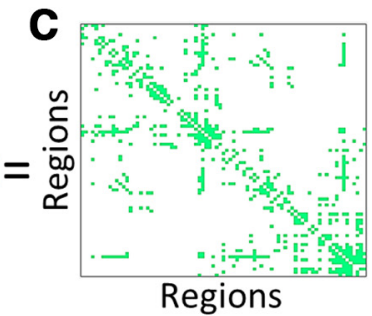

D

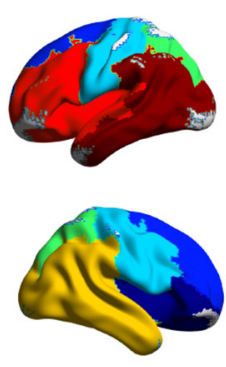

E
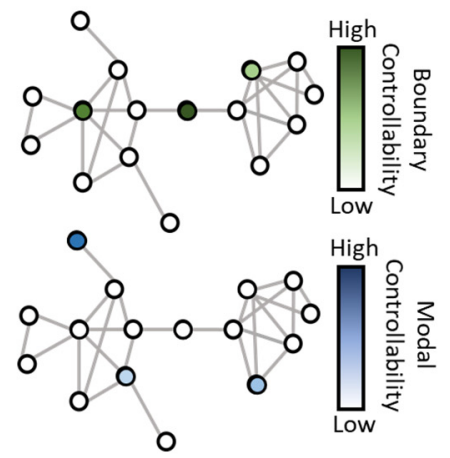

$\mathbf{F}$
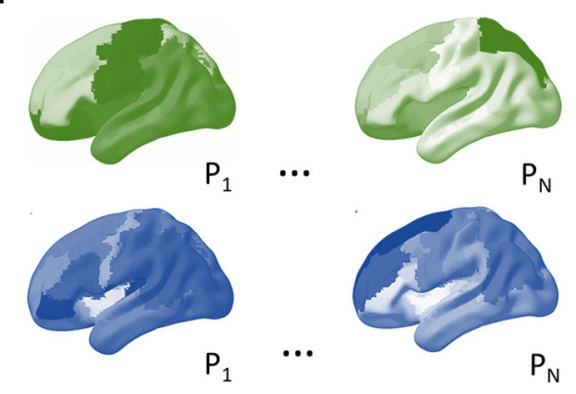

Figure 1. Overview of methods. $A$, cTBS was administered to each subject's pars triangularis. $B$, Diffusion tractography was computed for each subject. A cortical parcellation was registered to each individual's structural $\mathrm{T} 1$ image to identify anatomical divisions. C, A region $\times$ region structural adjacency matrix was constructed representing the streamline counts between pairs of regions binarized at a threshold retaining the top $10 \%$ strongest edge weights within subjects. $D$, A community detection algorithm was applied to identify an initial consensus partiation on the average network across subjects. $E$, Modal and boundary controllability were computed for each node (brain region) in the network for each individual. Each node received a rank representing its strength of control in the network heirarchy within the individual. $F$, Maps representing the variability in modal controllability (top) and boundary controllability (bottom). $P_{1 \ldots N}$ represent different participants. The relationship between controllability values at the LIFG stimulation site and task response times before and after stimulation were examined using mixed-effects models.

Although controlled language function is thought to be a network-level process (Doron et al., 2012, Chai et al., 2016, Fedorenko and Thompson-Schill, 2014), putative mechanisms of this process in the context of the brain's complex structural architecture remain unclear. Recent theoretical work in network control theory, an emerging area in engineering, provides one such mechanism. Network control theory (NCT) is the study of how to design control strategies for networked systems (Ruths and Ruths, 2014), in which a set of nodes are connected by edges, whereby a particular dynamic process occurs atop those edges. In the context of the brain, this suggests that brain regions (nodes) are predisposed to drive or modulate neurophysiological dynamics in a manner consistent with their specific topological role in brain networks constructed from white matter tractography. Variability in nodes' ability to drive the network into different trajectories may account for performance variability in controldemanding tasks ( $\mathrm{Gu}$ et al., 2015) and therefore the system's susceptibility to perturbation via TMS. However, a mechanistic network control role for the LIFG in language has not been tested experimentally.

Here, we tested whether NCT can identify potential mechanisms for language control. We investigated whether the theoretically predicted (i.e., mathematically derived) network control features of brain regions are related to performance on word retrieval tasks with varying cognitive control (and thus LIFG) demands (Hoffman et al., 2010, Whitney et al., 2011, Noonan et al., 2013, Ye and Zhou, 2009). In particular, we focused on openended semantic tasks, in which participants can choose one of several appropriate words to complete the task (Botvinick et al., 2001). This contrasts with a closed-ended task (number naming), which requires word retrieval but has only one correct response. We posit that language performance relates to the ability of the LIFG to control activity across human anatomical brain networks. In particular, we anticipate dissociable contributions of network control roles to open-language tasks (Jefferies and Lambon Ralph, 2006, Noonan et al., 2013, Hoffman et al., 2013, Jefferies, 2013) and closed-language tasks, which have different response retrieval and selection demands mediated by the pars triangularis (Hoffman et al., 2010).

To assess this view, we focus on two distinct network control features known as modal controllability (the theoretical ability of a node- here, a brain region- to drive a network into difficultto-reach states) and boundary controllability (the theoretical ability of a node to steer the system into states in which modules are either coupled or decoupled). Boundary controllability in the LIFG may represent intersystem coordination required for effective language production such as retrieving and selecting a single word in the face of competing, alternative words. Modal controllability in the LIFG may represent the recruitment of specific task-related states necessary for producing an accurate response (i.e., retrieving and selecting specific words according to varying task and/or response demands). We hypothesize that local inhibition via noninvasive brain stimulation (Benali et al., 2011, Huang et al., 2005) will allow us to distinguish network control roles corresponding to different cognitive roles of the pars triangularis (Hoffman et al., 2010) during open- and closed-language tasks.

\section{Materials and Methods}

\section{Overview of methods}

To address our hypotheses, we administered a form of noninvasive brain stimulation (transcranial magnetic stimulation; TMS) to a region within the LIFG (pars triangularis) in each of 19 healthy adult subjects between repeated administrations of two language tasks with open-ended selection demands and one number naming task with a single appropriate response for comparison. We also administered sham TMS to the vertex in each of nine healthy adult subjects between repeated administrations of the same tasks. Then, we constructed structural brain networks from diffusion spectrum imaging (DSI) data acquired for each subject (see Materials and Methods; Fig. 1A). Each network contained 111 brain 


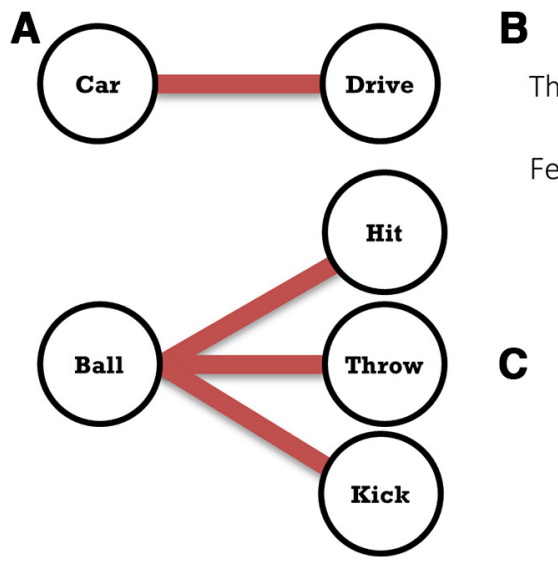

The girl walked the dog on the end of a

Few had the nerve to take the needed

scissors

Figure 2. Overview of tasks. $A$, High verbal selection demands are introduced when a cuing noun is associated with multiple appropriate words (here, verbs). Top, Example of a stimulus-response pairing with low selection demands. Bottom, Stimulusresponse pairing with high selection demands. $\boldsymbol{B}$, Example items from the sentence completion task. Participants were asked to provide an appropriate noun at the end of the sentence. Top, This item has a low selection demand because "leash" is easily and dominantly recalled in the context of this sentence. Bottom, This item has a high selection demand because several alternate words may be appropriate to complete the sentence. C, Example items from the verb generation task. Participants were asked to provide an appropriate verb associated with the noun. Left, This item has a low selection demand because "cut" is the most dominant verb associated with "scissors." Right, This item has a high selection demand because several verbs are highly associated with the mouth, such as "eat," "talk," and "kiss."

regions defined by the Lausanne anatomical parcellation and cerebellum (Fig. $1 B$ ) and each pair of regions was connected by an edge weighted by the number of streamlines linking those regions (Fig. 1C). We defined a simplified model of brain dynamics and simulated network control to quantify modal and boundary controllability (Fig. 1D).

\section{Subjects}

Twenty-eight healthy individuals ( $m e a n$ age $=25.4, \mathrm{SD}=4.5,16$ female) were scanned on a 3 T Prisma scanner at the University of Pennsylvania. All procedures were approved in a convened review by the University of Pennsylvania's Institutional Review Board and were performed in accordance with the guidelines of the Institutional Review Board/Human Subjects Committee, University of Pennsylvania. All participants volunteered with informed consent in writing before data collection.

\section{Neuroimaging: diffusion tractography}

DSIs were acquired for a total of 28 subjects along with a T1-weighted anatomical scan at each scanning session. We followed a parallel strategy for data acquisition and construction of streamline adjacency matrices as in previous work applying network controllability statistics in human diffusion imaging networks (Gu et al., 2015). DSI scans sampled 257 directions using a Q5 half-shell acquisition scheme with a maximum $b$-value of 5000 and an isotropic voxel size of $2.4 \mathrm{~mm}$. We used an axial acquisition with the following parameters: $\mathrm{TR}=5 \mathrm{~s}, \mathrm{TE}=138 \mathrm{~ms}, 52$ slices, FOV: 231, 231, $125 \mathrm{~mm}$.

DSI data were reconstructed in DSI Studio (dsi-studio.labsolver.org) using q-space diffeomorphic reconstruction (QSDR) (Yeh et al., 2011). QSDR first reconstructs diffusion-weighted images in native space and computes the quantitative anisotropy (QA) in each voxel. These QA values are used to warp the brain to a template QA volume in Montreal Neurological Institute (MNI) space using the statistical parametric mapping nonlinear registration algorithm. Once in MNI space, spin density functions were again reconstructed with a mean diffusion distance of $1.25 \mathrm{~mm}$ using 3 fiber orientations per voxel. Fiber tracking was performed in DSI Studio with an angular cutoff of $35^{\circ}$, step size of $1.0 \mathrm{~mm}$, minimum length of $10 \mathrm{~mm}$, spin density function smoothing of 0.0 , maximum length of $400 \mathrm{~mm}$, and a QA threshold determined by diffusion-weighted imaging signal in the colony-stimulating factor. Deterministic fiber tracking using a modified FACT algorithm was performed until 1,000,000 streamlines were reconstructed for each individual. DSI Studio placed starting points within seeding "voxels" at subvoxel mouth

resolution. The actual seeding points were determined randomly and uniformly within the voxels. DSI Studio used a deterministic random generator to place the seeds, so the seeding sequence was both deterministic and random. These features ensured that the tracking result is reproducible using the same tracking parameters. DSI Studio drew a point within the voxel range using a uniform distribution. The point was then used as the starting point within the selected voxel.

Anatomical (T1) scans were segmented using FreeSurfer (Fischl, 2012) and parcellated using the connectome mapping toolkit (Cammoun et al., 2012). A parcellation scheme including $n=111$ regions was registered to the B0 volume from each subject's DSI data. The B0 to MNI voxel mapping produced via QSDR was used to map region labels from native space to MNI coordinates. To extend region labels through the gray-white matter interface, the atlas was dilated by $4 \mathrm{~mm}$ (Cieslak and Grafton, 2014). Dilation was accomplished by filling nonlabeled voxels with the statistical mode of their neighbors' labels. In the event of a tie, one of the modes was arbitrarily selected. Each streamline was labeled according to its terminal region pair. From these data, we constructed a structural connectivity matrix, $A$, the element of which, $A_{\mathrm{ij}}$, represented the number of streamlines connecting different regions divided by the sum of volumes for regions $i$ and $j$ (Hagmann et al., 2008). There are numerous free parameters in diffusion tractography, image parcellation, and graph representations of anatomical connectivity (e.g., weighted vs binarized — or unweighted — graphs). In the current sample, we found that values computed in binarized networks that were defined from the top $10 \%$ strongest weights within subjects yielded fully stable and uniformly distributed controllability statistics across subjects over 100 separate iterations of the controllability scripts, whereas the statistics computed from the weighted networks tended to exhibit higher skewness or non-normal distributions. Therefore, we retained the binarized controllability statistics for our primary analyses.

\section{Cognitive testing}

Participants performed two open-ended language tasks and one closedended number naming task (Fig. 2).

The language tasks included a verb generation task and a sentence completion task (Snyder and Munakata, 2008). For the verb generation task, subjects were instructed to generate the first verb that came to mind when presented with a noun stimulus (e.g., "cat"). The verb could be either something the noun does (e.g., "meow") or something you do with it (e.g., "feed"). Response times (RTs) were collected from the onset of the noun cue to the onset of the verb response. For the sentence completion task, participants were presented with a sentence, such as "They left the dirty dishes in the __." and were instructed to generate a single word that appropriately completes the sentence, such as "sink". Words in the sentences were presented serially in $1 \mathrm{~s}$ segments consisting of 1 or 2 words. RTs were computed as the latency between the onset of the last segment, which always contained a two-word segment (i.e., a word and an underline) and the onset of the participant's response. For all items in the sentence completion task, items in the high versus low selection demand conditions were matched on retrieval demands (association strength; Snyder and Munakata, 2008). For both language tasks, each trial began with the presentation of a fixation point $(+)$ for $500 \mathrm{~ms}$, followed by the presentation of the target stimulus, which remained on the screen for $10 \mathrm{~s}$ until the subject made a response. Subjects were given an example and five practice trials in the first administration of each language task (i.e., before TMS) and were reminded of the instructions before performing the task a second time (i.e., after TMS). In each of the before and after TMS conditions, subjects completed 50 trials for a total of 100 trials. 
The items for the verb generation task were identical to those used in Snyder et al. (2011) and the items for the sentence completion task were those from Snyder et al. (2014). The difficulty of items was sampled to cover a distribution of values computed via latent semantic analysis (LSA) applied to corpus data. In particular, items were sampled to represent a range of LSA entropy and LSA association strength (Snyder and Munakata, 2008), which represent the selection and retrieval demands of each item, respectively (Snyder and Munakata, 2008). An LSA association value of 0 means that the cue word or sentence is not strongly associated with any word in particular, whereas a value of 1 means that the cue word or sentence is strongly associated with at least one word, implying that it is easy to retrieve. An LSA entropy value of 0 indicates that the word is not related to any words, whereas higher values indicate higher relatedness to many words, which theoretically increases competition among appropriate words (Snyder and Munakata, 2008).

The comparison task requiring closed-ended responses was a number naming task in which participants produced the English names for strings of Arabic numerals presented on the screen. On each trial, a randomized number (from tens of thousands to millions; e.g., 56395, 614592, 7246856) was presented in black text on a white background. The numbers were uniformly distributed over three lengths (17 per length for each task administration). The position of items on the screen was randomized between the center, left, and right of the screen to reduce the availability of visual cues to number length and syntax (Krieger-Redwood and Jefferies, 2014). RTs were collected from the onset of the stimulus presentations to the onset of the subject's response. The number appeared in gray after the detection of a response (i.e., voice key trigger) and remained on the screen thereafter to reduce the working memory demands required for remembering the digit string. At the start of the experiment, subjects performed 50 trials of the number naming task to account for initial learning effects (Krieger-Redwood and Jefferies, 2014). Before performing the task for the first time, subjects were given an example and five practice trials and were later reminded of the instructions before performing the task a second time (i.e., before TMS) and a third time (i.e., after TMS). In each of the before and after TMS conditions, subjects completed 51 trials for a total of 102 experimental trials.

Verbal responses for all tasks were collected from a computer headset microphone. The microphone was calibrated to reduce sensitivity to environment background noise before the collection of data for each session such that the recording software was not triggered without clear verbalizations. List order (before or after TMS) was counterbalanced across participants. Item presentation order within each task was fully randomized across participants.

\section{TMS}

The Brainsight system (Rogue Research) was used to coregister MRI data with the location of the subject and the TMS coil. The stimulation site was defined as the posterior extent of the pars triangularis in each individual subject's registered T1 image. A Magstim Super Rapid ${ }^{2}$ Plus ${ }^{1}$ stimulator was used to deliver continuous theta-burst stimulation (cTBS) via a $70-\mathrm{mm}$ diameter figure eight coil. To calibrate the intensity of stimulation, cTBS was delivered at $80 \%$ of each participant's active motor threshold (Huang et al., 2005). Each participant's threshold was determined before the start of the experimental session using a standard up-down staircase procedure with stimulation to the motor cortex (M1). In the sham condition, the coil was held against the head at a $90^{\circ}$ angle at the subject's vertex to introduce a degree of induced electrical stimulation of the scalp. We administered sham at vertex to reduce the possibility that subjects could see the orientation of the coil in the sham condition because subjects were not naive to TMS.

\section{Mathematical models}

\section{Network control theory}

We follow a previous application of network control theory in diffusion weighted imaging data as the basis for our examination of controllability and cognitive control. We briefly describe the mathematical basis for the approach taken here. For a full discussion of structural network controllability in the context of diffusion-weighted imaging networks, see Gu et al. (2015). For a full discussion of the mathematical basis for structural network controllability, see Liu et al. (2011), Ruths and Ruths (2014), and Pasqualetti et al. (2014).

Our ability to understand neural systems is fundamentally related to our ability to control them (Schiff, 2012). Network control theory is a branch of traditional control theory in engineering that examines how to control a system based on the pattern of links between its components, and based on a model of the system's dynamics. Here, we interpret the word "control" to mean perturbing communication in an anatomical brain network. To apply a network control perspective, we require knowledge of the network connectivity linking system components and knowledge regarding how system components function (i.e., their dynamics), rather than simply a descriptive statistics of the network's architecture. In contrast to traditional graph theory, network control theory offers mechanistic predictors of network dynamics. The use of mechanistic models allows us enrich descriptive approaches to examine the human connectome (Medaglia et al., 2015) with statistics that explicitly include a dynamic model.

Mathematically, we can study the controllability of a networked system by defining a network represented by the graph $G=(V, E)$, where $V$ and $E$ are the vertex and edge sets, respectively. Let $a_{\mathrm{ij}}$ be the weight associated with the edge $(i, j) \in \varepsilon$ and define the weighted adjacency matrix of $G$ as $A=\left[a_{\mathrm{ij}}\right]$, where $a_{\mathrm{ij}}=0$ whenever $(i, j) \notin \varepsilon$. We associate a real value (state) with each node, collect the node states into a vector (network state) and define the map $x: \mathbb{N}_{\geq 0} \rightarrow \mathbb{R}^{n}$ to describe the evolution (network dynamics) of the network state over time. Given the network and node dynamics, we can use network control theory to examine quantitatively how the network structure relates to the types of control that nodes can exert.

\section{Dynamic model of neural processes}

We begin with an analogous approach to prior work (Gu et al., 2015). We define structural brain networks by subdividing the entire brain into anatomically distinct brain areas (network nodes) in a commonly used anatomical atlas (Hagmann et al., 2008). Consistent with prior work (Bassett et al., 2011, Hermundstad et al., 2013, 2014, Gu et al., 2015), we connect nodes by the number of white matter streamlines identified by a commonly used deterministic tractography algorithm (for details on the tractography implementation, see Cieslak and Grafton, 2014). This procedure results in sparse, weighted, undirected structural brain networks for each subject. Properties of this network include high clustering, short path length, and strong modularity, consistent with prior studies of similar network data (Bassett et al., 2011, Hagmann et al., 2008). The definition of structural brain networks based on tractography data in humans follows from our primary hypothesis that control features of neural dynamics are in part determined by the structural organization of the brain's white matter tracts.

To define the dynamics of neural processes, we draw on prior models linking structural brain networks to resting-state functional dynamics (Honey et al., 2009, 2010, Abdelnour et al., 2014). Although neural activity evolves through neural circuits as a collection of nonlinear dynamic processes, these prior studies have demonstrated that a significant amount of variance in neural dynamics as measured by resting-state fMRI can be predicted from simplified linear models. Based on this literature, we use a simplified noise-free linear discrete-time and time-invariant network model as follows:

$$
\mathbf{x}(t+1)=\mathbf{A x}(t)+\mathbf{B}_{K} \mathbf{u}_{K}(t)
$$

where $\mathbf{x}: \mathbb{R}_{\geq 0} \rightarrow \mathbb{R}^{N}$ describes the state (e.g., a measure of the electrical charge, oxygen level, or firing rate) of brain regions over time and $\mathbf{A} \in \mathbb{R}^{N \times N}$ is a symmetric and weighted adjacency matrix. In this case, we construct a weighted adjacency matrix with elements that indicate the number of white matter streamlines connecting two different brain regions, denoted here as $i$ and $j$, and we stabilize this matrix by dividing by the mean edge weight. Although the model used above is a discrete-time system, we find that the controllability Gramian is statistically similar to that obtained in a continuous-time system (Gu et al., 2015). 
The diagonal elements of the matrix $A$ satisfy $A_{\mathrm{ii}}=0$. The input matrix $B_{\kappa}$ identifies the control points $\kappa$ in the brain, where $K=\left\{k_{1}, \ldots, k_{m}\right\}$ and the following:

$$
B_{K}=\left[\begin{array}{lll}
e_{k_{1}} & \ldots & e_{k_{m}}
\end{array}\right]
$$

and $e_{\mathrm{i}}$ denotes the $i$-th canonical vector of dimension $N$. The input $\mathbf{u}_{K}: \mathbb{R}_{\geq 0} \rightarrow \mathbb{R}^{m}$ denotes the control energy.

\section{Network controllability}

To study the ability of a certain brain region to influence other regions in arbitrary ways, we adopt the control theoretic notion of controllability. Controllability of a dynamical system refers to the possibility of driving the state of a dynamical system to a specific target state by means of an external control input (Kalman et al., 1963). In the current study, we follow the procedures applied in Gu et al. (2015) and focus on two network controllability statistics: boundary and modal controllability. It is critical to note that the controllability statistics examined here are based on linear discrete time dynamics, but they have also been extended recently to nonlinear models of dynamics. In simulation studies, linear controllabilty statistics were related to predicted effects in dynamics simulated using Wilson-Cowan (Muldoon et al., 2016) oscillators in anatomical brain networks. In addition, they predicted topological changes in network dynamics simulated using Kuramoto oscillators (Tiberi et al., 2017). Therefore, we focus on mathematically well defined linear control statistics due to their parsimony and pragmatic utility in applied contexts such as neuromodulation research.

Boundary controllability. Boundary controllability, a metric developed in network control theory, quantifies the role of a network node in controlling dynamics between modules in hierarchical modular networks (Pasqualetti et al., 2014). Boundary controllability identifies brain areas that can steer the system into states where different cognitive systems are either coupled or decoupled. A region's boundary controllability describes its theoretical ability to regulate the extent to which it can drive major networks to increase or decrease communication with one another. High boundary controllers are conceptually akin to the "gatekeepers" of communication between major brain networks. Here, we apply a similar approach to that used previously (Gu et al., 2015) to quantify boundary controllability in our diffusion tractography networks and associate controllability variability with cognitive performance. Specifically, we partition the brain into modules by maximizing the modularity quality function (Newman, 2006) using a Louvain-like (Blondel et al., 2008) locally greedy algorithm (Jutla et al., 2011). Because the modularity quality function has many near degeneracies, we perform the optimization algorithm multiple times (Good et al., 2010). We observed that the mean partition similarity was high and the variance of the partition similarity was low for a value of $\gamma$ at 1.6 (mean $z$-Rand score $=58.3, \mathrm{SD}=2.8$ ), which is within the range of stable partitions found in prior analyses of DSI data (Gu et al., 2015). We therefore used the consensus partition at $\gamma=1.6$ for the remainder of the analysis in this study. High-ranking boundary controllers are identified as the highest ranking set of boundary regions and remaining boundary regions are found within modules in the network.

Modal controllability. Modal controllability refers to the ability of a node to control each evolutionary mode of a dynamical network (Hamdan and Nayfeh, 1989) and can be used to identify the least controllable state from a set of control nodes. Modal controllability is computed from the eigenvector matrix $V=\left[v_{\mathrm{ij}}\right]$ of the network adjacency matrix $A$. By extension from the PBH test (Kailath, 1980), if the entry $v_{\mathrm{ij}}$ is small, then the $j$-th mode is poorly controllable from node $i$. As described previously (Pasqualetti et al., 2014), we define $\phi_{i}=\sum_{j=1}^{N}\left(1-\lambda_{j}^{2}(A)\right) v_{i j}^{2}$ as a scaled measure of the controllability of all $N$ modes $\lambda_{1}(A), \ldots, \lambda_{N}(A)$ from the brain region $i$. Regions with high modal controllability are able to control all the dynamic configurations of the network, and thus to drive the dynamics toward hard-to-reach configurations. A brain region's modal controllability describes its theoretical ability to drive the brain into states that are difficult to reach. Dynamically, these states typically involve the activation of a few specific regions in the network. High modal controllers are conceptually akin to dynamic "specialists" driving specific, otherwise unachievable, states.
Node centrality and controllability. There is a key distinction between the controllability statistics and more commonly applied node centrality statistics. Although more common node centrality measures are used to make inferences about the role of brain regions in information processing (Rubinov and Sporns, 2010), network controllability statistics explicitly encode dynamics. This means that they support a theoretical inference about the role of brain regions in controlling states. One crucial value of applying network control theory in neural stimulation is that it paves the way for explicit connections to systems engineering and control theory, extending past passive analysis of network topology.

In the current data, boundary controllability is not significantly correlated with node degree $(r=-0.25, p=0.17)$ or closeness centrality $(r=0.27, p=0.15)$ and is moderately correlated with betweenness centrality $\left(r=0.62, p=2.98 \times 10^{-4}\right)$. Modal controllability in the LIFG is negatively but imperfectly correlated with node degree (the sum of edges emanating from LIFG; $\left.R=-0.94, p=6.62 \times 10^{-14}\right)$ is correlated with node betweenness centrality (the number of shortest paths through the network that pass through the LIFG; $r=-0.50, p=$ 0.006 ) and not correlated with node closeness centrality (the sum of the length of shortest paths between the LIFG and all other regions; $r=$ $-0.29, p=0.13$ ).

These findings indicate that, in addition to supporting a theoretical inference in network control mediated by the LIFG, modal controllability replicates a strong negative relationship with node degree (Gu et al., 2015) and both control statistics are moderately related to centrality among network paths. However, each type of controllability does not completely overlap with basic measures of centrality calculated from static network topology.

\section{Statistical analysis: examining the relationship among controllability, cognition, and TMS effects}

Analyses were conducted using multilevel modeling with maximumlikelihood estimation (Baayen et al., 2008) implemented in the lme4 version 1.1-9 (Bates et al., 2015) package of $\mathrm{R}$ version 3.2.1 (R Core Team, 2016). This technique allows for a classical regression analysis to be performed on repeated-measures data by accounting for the nonindependence of observations collected from each participant in a withinsubjects design without resorting to computing separate regression equations for each subject (Lorch and Myers, 1990, Baayen et al., 2008, Baayen, 2008). Multilevel modeling also accounts for violations of the sphericity assumption by modeling heteroskedasticity in the data when necessary, improving statistical power over other methods commonly used for analyzing repeated-measures data. We excluded from analyses trials on which participants responded incorrectly (i.e., semantic and paraphasic errors, hesitations, or false starts) and experimenter error/ equipment failures (such as false triggers for voice recording), constituting a mean of $6.66 \%$ and $3.68 \%$ of all trials, respectively. RTs in open- and closed-ended tasks were log-transformed due to non-normal distribution of raw RTs.

To independently isolate relationships between open and closed task performance as a function of boundary or modal controllability and their potential modulation via TMS, we computed four separate mixed models (i.e., two each assessing boundary and modal controllability on openand closed-task performance). In all models, fixed effects included the within-subjects factor of session (before vs after stimulation), the between-subjects factors of TMS (active vs sham), and continuous variables reflecting either boundary or modal controllability. For each controllability measure (boundary and modal), separate models were run with log-transformed RTs for the open-ended (i.e., verb generation and sentence completion) and closed-ended (i.e., number naming) tasks as the dependent variable. In all analyses, we attempted to fit models using maximal random effect structures because this method increases model generalizability (Barr et al., 2013). Random effect structures were simplified as required for model convergence to reduce the likelihood of model overfitting. Final models reported below included by-participant random intercepts and by-participant random slopes for the effects of trial within task per session, which coled for overall differences in mean RTs and fatigue across the course of each task within each experimental session. Moreover, the inclusion of by-participants random effects accounts 
for any estimation bias due to unequal and small sample sizes (Bell et al., 2014). None of the final models reported produced convergence warnings indicative of model overfitting. In the Results section, "high" and "low" values for high and low boundary and modal controllability are with respect to a median split to make the interpretation of the directions of the effects clearer.

\section{Code availability}

Code for calculating the controllability statistics examined here is available on Github (https://github.com/nangongwubu/NetworkControllability-Diagnostics).

\section{Results}

For context, we computed the mean and SD of the ranked boundary and modal controllability values for all regions across the brain. As illustrated in Figure 3, LIFG boundary controllability values in our sample tended to lie in the middle range with moderate variability compared with other brain regions, whereas modal controllability in the LIFG tended to have higher values with low variability compared with other regions. To be sure that boundary and modal controllability capture unique topological control roles in the context of the brain's anatomical network, we assessed the relationship between these two metrics across subjects in the current sample. Here, modal and boundary controllability in the LIFG were weakly correlated $(r=0.10, p=0.61)$, sharing only $\sim 1 \%$ variance. This observation suggests that boundary and modal controllability theoretically contribute to distinct network control processes. We therefore present the results in separate models to isolate the effects associated with each network control role in the LIFG.

We computed four multilevel regression models for the relationships between modal and boundary controllability at the LIFG and performance on the open- and closed-demands language tasks before and after active versus sham stimulation. In our interpretation of the results, we restrict our focus to cTBS-related findings indicating that inhibitory stimulation of the LIFG modulates performance (i.e., before vs after differences) compared with sham stimulation and that the degree to which the LIFG specializes in boundary or modal controllability predicts responsiveness to TMS. The rationale for this focus is twofold. First, performance changes before versus after stimulation will reveal whether receiving active stimulation alters potential practice-related effects as established with sham stimulation. Second, demonstrating that active stimulation to the LIFG (compared with sham stimulation) affects performance based on the LIFG's control role in the network provides a strong test of this region's involvement in language tasks with open and closed demands, which in turn sheds light on the potential mechanisms of LIFG-mediated control.

Open-ended language task performance, TMS, and boundary and modal controllability

The model assessing the effect of boundary control on open-ended tasks revealed that cTBS of the LIFG modulated performance as a function of boundary controllability. Specifically, in the sham group, high boundary controllability was associated with longer RTs (henceforth reported as log-transformed values) after sham stimulation [i.e., faster RTs before vs after sham; mean (SE) = $7.29(0.11)$ vs $7.48(0.01)$, respectively], demonstrating a practicerelated interference effect. After cTBS, the practice-related interference effect was eliminated [i.e., similar RTs before and after cTBS; mean $(\mathrm{SE})=7.17(0.04)$ and $7.18(0.05)$, respectively] This suggests that inhibitory TMS mitigates interference when the LIFG is more heavily involved in an integrative role in the brain (Fig. 4, Table 1).

Modal controllability was not associated with changes in openended task performance as a function of TMS (Fig. 5, Table 2).

\section{Closed-ended language task performance, TMS, and boundary and modal controllability}

Boundary controllability was not associated with changes in closed-ended task performance as a function of TMS (Fig. 6, Table 3).

In contrast, modal controllability within the LIFG was associated with TMS-induced changes in closed-ended task performance. Modest practice-related facilitation was observed in all subjects and was relatively enhanced for individuals with low modal controllability after TMS [mean (SE) RTs before vs after $=$ $7.11(0.07)$ vs $6.98(0.06)$, respectively] relative to sham [mean (SE) RTs before vs after $=7.02(0.10)$ vs 6.97 (0.09), respectively]. This suggests that inhibitory TMS enhances practice effects when denser local anatomical connections (associated with lower modal controllability) place increased processing demands on the LIFG when the task requires selection of a specific response (Fig. 7, Table 4). 


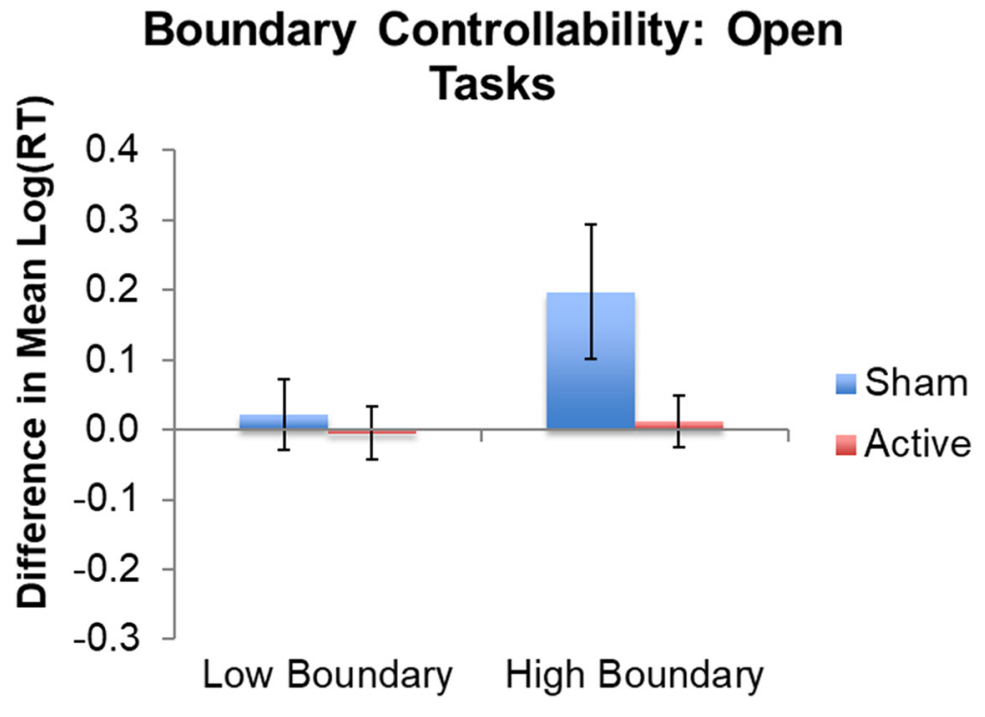

Figure 4. TMS effects in the significant interaction between TMS and boundary controllability in open language tasks. "High" and "low" represent boundary controllability ranked values above and below the median, respectively, for the sample for illustration. Error bars indicate SE of the differences in mean log-transformed RTs. Lower values on the $y$-axis represent faster responses in the second session relative to the first session. See Table 1 for full model results. Note that, for transparency, the mean and SE of the log-transformed RTs do not represent model-adjusted values.

Table 1. Mixed-effects model for the effects of boundary controllability, session, and TMS in the open tasks

\begin{tabular}{lrrrc}
\hline & Estimate & \multicolumn{1}{l}{ SE } & \multicolumn{1}{c}{$t$-value } & $p$ \\
\hline Intercept & 7.370 & 0.252 & 29.257 & $<0.001^{* * *}$ \\
Session & -0.109 & 0.097 & -1.124 & 0.261 \\
ActiveSham & -0.620 & 0.322 & -1.928 & 0.063 \\
Boundary & -0.002 & 0.003 & -0.775 & 0.444 \\
Session*Boundary & -0.001 & 0.001 & 1.116 & 0.264 \\
Session*ActiveSham & 0.412 & 0.124 & 3.328 & $<0.001^{* * *}$ \\
Boundary*ActiveSham & 0.009 & 0.004 & 2.233 & $<0.033^{*}$ \\
Boundary*ActiveSham*Session & -0.007 & 0.002 & -4.124 & $<0.001^{* * *}$ \\
\hline
\end{tabular}

ActiveSham, Active TMS effect relative to sham; boundary, continuous boundary controllability effect; session, effect of the second relative to the first session.

${ }^{*} p<0.05 ;{ }^{* *} p<0.01 ;{ }^{* * *} p<0.001$.

\section{Summary of LIFG}

controllability results

To summarize, boundary controllability, representing the theoretical ability to integrate and segregate network communication, may represent a control demand specific to open-ended language tasks, namely the need to retrieve and select a single word in the face of competing, alternative words when many responses may be equally appropriate (given a cue). This is suggested by our findings that boundary controllability within the LIFG predicted individuals' responsiveness to TMS in open-ended (but not the closedended) language tasks. In contrast, modal controllability, the ability to steer the network in difficult-to-reach states, may represent a control process important for responding in the closed-ended language task; that is, the need to select specific words according to task demands. The lack of a TMS effect related to boundary controllability and performance on the closed-ended task suggests that the LIFG's ability to integrate and segregate communication between brain networks may not play an important role when the task demands require retrieving and selecting a single, correct representation rather than one from among several possible acceptable alternatives. Likewise, the observation that modal controllability did not relate to TMS effects on open-ended tasks could suggest that the LIFG's role in achieving difficult-to-reach states does not play an important role in selection words from competing alternatives. Taken together, the analyses reveal dissociable control roles of the LIFG relevant for understanding this region's involvement in word selection under open- and closed-ended response demands by virtue of how individuals respond to exogenous brain stimulation.

\section{Modal Controllability: Open Tasks}

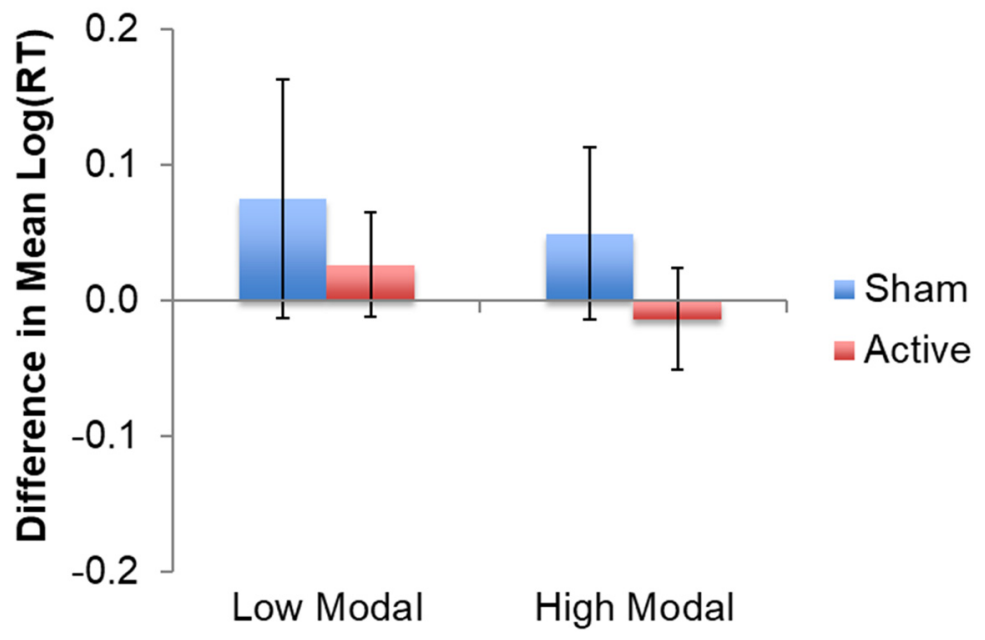

Figure 5. TMS effects in the significant interaction between TMS and modal controllability in open language tasks. "High" and "low" represent modal controllability ranked values above and below the median, respectively, for the sample for illustration. Error bars indicate SE of the differences in mean log-transformed RTs. Lower values on the $y$-axis represent faster responses in the second session relative to the first session. See Table 2 for full model results. Note that, for transparency, the mean and SE log-transformed RTs do not represent model-adjusted values.
Control comparison examining TMS effects and boundary and modal control within the left lateral occipital cortex

As a control analysis for LIFG regionspecific effects within the connectome, we replicated our multilevel regression analyses using the boundary and modal controllability ranked values from the left lateral occipital region (Fig. 3). In a functional context, the occipital lobe contributes "bottom-up" processing in the context of the "top-down" control processes thought to be mediated by the LIFG. Furthermore, the left lateral occipital region constitutes a strong statistical control because variability in the rank network control values in the left lateral occipital lobe and LIFG (pars triangularis) region were comparable for boundary controllability (i.e., $\mathrm{SD}=15.3$ and 18.7 , respectively) and modal controllability (i.e., SD $=6.4$ and 11.9 , respectively). These analyses indicate that the influence of TMS on open 
tasks relative to sham interacts with occipital cortex boundary controllability (specific effect: $p=0.002$; Table 5), but not modal controllability ( specific effect: $p=0.28$ ).

However, the influence of TMS on the closed tasks relative to sham also does not interact with occipital cortex boundary controllability (specific effect: $p=0.29$ ) or modal controllability (specific effect: $p=0.89$ ). Overall, these observations indicate that the TMS effects on open-ended task performance related to boundary controllability of the "top-down" region at the site of stimulation also apply to an unstimulated "bottom-up" region within the larger left hemispheric language network. The fact that the model reveals a pattern in the same direction (i.e., practicerelated interference observed with increasing boundary controllability is eliminated with active TMS) suggests that inferior frontal control mechanisms and the posterior visual system's role in integrating and segregating functional communication between other network modules across the brain contribute jointly to performance. In contrast, TMS effects on closed-task performance as it relates to modal controllability are specific to the "top-down" site of stimulation, which may indicate that the local network processing at LIFG measured by modal controllability dissociates from the network-wide processes related to boundary controllability.

\section{Discussion}

Here, we examined the hypothesis that network controllability in the LIFG is related to language performance in tasks with open- and closed-ended response demands. We explicitly tested this hypothesis by linking variability in the vulnerability of controlled language function to perturbation by TMS to LIFG controllability. In this study, we integrated two separately developing theoretical frameworks from cognitive neuroscience and emerging applications of control theory to human brain networks ( $\mathrm{Gu}$ et al., 2015). In cognitive neuroscience, the LIFG is identified as a site that mediates controlled language function; however, the mechanisms by which the LIFG executes this role in brain networks are unknown. Network control theory is postulated to be a useful framework to understand the organization for human cognitive control and performance variability based on the role of anatomical regions in the structural connectome (Gu et al., 2015, Tang et al., 2017).

To test this experimentally, we constructed structural brain networks from DSI data acquired in 28 healthy adult individuals and administered inhibitory TMS $(n=19)$ or sham stimulation $(n=9)$ between two repetitions of language tasks that differ in open- and closed- response demands. We anticipated that dissociable network control roles in the pars triangularis of the LIFG, boundary and modal controllability, would be associated with distinct TMS-induced performance changes in the tasks with open and closed demands, respectively. Our results suggest that such a dissociation exists. Specifically, boundary controllability was associated with TMS effects on word selection under openended response demands, whereas modal controllability was associated with TMS effects on word selection under closed-ended response demands.
Table 2. Mixed-effects model for the effects of modal controllability, session, and TMS in the open tasks

\begin{tabular}{lrlrc}
\hline & Estimate & \multicolumn{1}{l}{ SE } & \multicolumn{1}{c}{$t$-value } & \multicolumn{1}{l}{$p$} \\
\hline Intercept & 6.938 & 0.292 & 23.730 & $<0.001^{* * *}$ \\
Session & -0.003 & 0.112 & -0.027 & 0.978 \\
ActiveSham & 0.025 & 0.531 & 0.046 & 0.963 \\
Modal & 0.005 & 0.006 & 0.822 & 0.418 \\
Session*Modal & $<0.001$ & 0.002 & 0.010 & 0.992 \\
Session*ActiveSham & -0.175 & 0.215 & -0.812 & 0.417 \\
Modal*ActiveSham & $<0.001$ & 0.010 & 0.025 & 0.980 \\
Modal*ActiveSham*Session & 0.002 & 0.004 & 0.546 & 0.585 \\
\hline
\end{tabular}

ActiveSham, Active TMS effect relative to sham; modal, continuous modal controllability effect; session, effect of the second relative to the first session.

${ }^{*} p<0.05 ;{ }^{* *} p<0.01 ;{ }^{* * *} p<0.001$.

\section{Boundary Controllability: Closed} Task

Figure 6. TMS effects in the significant interaction between TMS and boundary controllability during the closed language task. "High" and "low" represent boundary controllability ranked values above and below the median, respectively, for the sample for illustration. Error bars indicate SE of the differences in mean log-transformed RTs. Lower values on the $y$-axis represent faster responses in the second session relative to the first session. See Table 3 for full model results. Note that, for transparency, the mean and SE of the log-transformed RTs do not represent model-adjusted values.

Table 3. Mixed-effects model for the effects of boundary controllability, session, and TMS in the closed task

\begin{tabular}{lrlrc}
\hline & Estimate & \multicolumn{1}{l}{ SE } & \multicolumn{1}{c}{$t$-value } & \multicolumn{1}{l}{$p$} \\
\hline Intercept & 7.021 & 0.125 & 56.182 & $<0.001^{* * *}$ \\
Session & 0.081 & 0.089 & 0.913 & 0.361 \\
ActiveSham & -0.596 & 0.245 & -1.594 & 0.122 \\
Boundary & -0.008 & 0.004 & -2.174 & $0.038^{*}$ \\
Session*Boundary & $<0.001$ & 0.001 & -0.080 & 0.937 \\
Session*ActiveSham & -0.116 & 0.113 & -1.028 & 0.304 \\
Boundary*ActiveSham & 0.008 & 0.005 & 1.555 & 0.131 \\
Boundary*ActiveSham*Session & 0.002 & 0.001 & 1.045 & 0.296 \\
\hline
\end{tabular}

ActiveSham, Active TMS effect relative to sham; boundary, continuous boundary controllability effect; session, effect of the second relative to the first session.

${ }^{*} p<0.05 ;{ }^{* *} p<0.01 ;{ }^{* * *} p<0.001$.

Importantly, by evaluating LIFG network control roles in the context of performance changes after active compared with sham stimulation, the current study provides insight into the mechanisms by which the LIFG supports controlled language function. In particular, our finding that boundary controllability within the LIFG was associated with TMS effects in the open-ended, but not closed-ended, language tasks suggests that the integration and segregation of intermodular communication may be partic- 


\section{Modal Controllability: Closed Task}

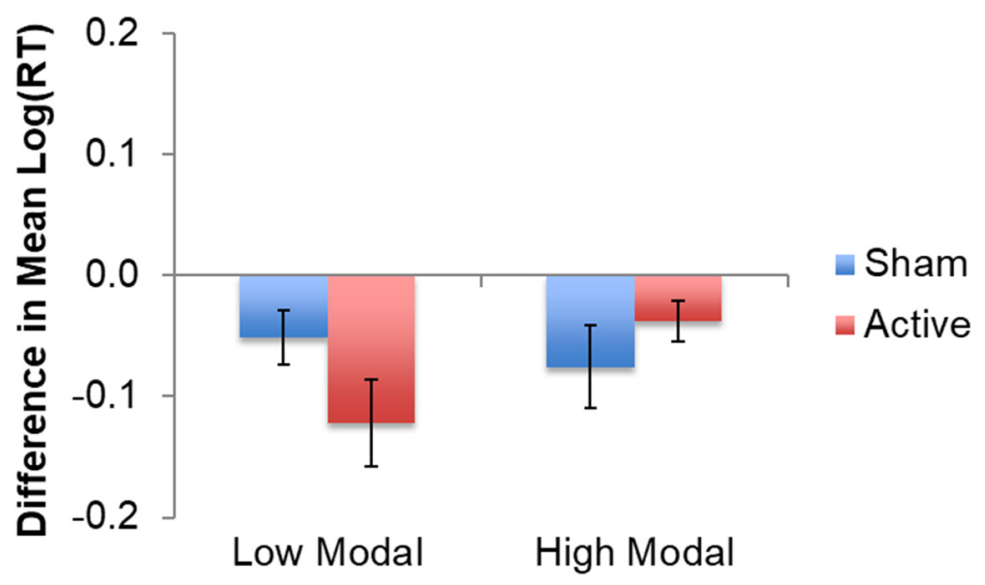

Figure 7. TMS effects in the significant interaction between TMS and modal controllability during the closed language task. "High" and "low" represent modal controllability ranked values above and below the median, respectively, for the sample for illustration. Error bars indicate SE of the differences in mean log-transformed RTs. Lower values on the $y$-axis represent faster responses in the second session relative to the first session. See Table 4 for full model results. Note that, for transparency, the mean and SE log-transformed RTs do not represent model-adjusted values.

Table 4. Mixed-effects model for the effects of modal controllability, session, and TMS during the closed task

\begin{tabular}{lrlrc}
\hline & Estimate & \multicolumn{1}{l}{ SE } & \multicolumn{1}{c}{$t$-value } & \multicolumn{1}{l}{$p$} \\
\hline Intercept & 7.079 & 0.357 & 19.820 & $<0.001^{* * *}$ \\
Session & 0.311 & 0.104 & 2.977 & $0.003^{* *}$ \\
ActiveSham & -0.185 & 0.647 & -0.286 & 0.777 \\
Modal & -0.002 & 0.007 & -0.306 & 0.762 \\
Session*Modal & -0.005 & 0.002 & -2.283 & $0.023^{* *}$ \\
Session*ActiveSham & -0.365 & 0.181 & -2.013 & $0.044^{*}$ \\
Modal $^{*}$ ActiveSham & 0.004 & 0.012 & 0.322 & 0.749 \\
Modal $^{*}$ ActiveSham*Session & 0.007 & 0.003 & 1.988 & $0.047^{*}$
\end{tabular}

ActiveSham, Active TMS effect relative to sham; modal, modal controllability effect; session, effect of the second relative to the first session.

${ }^{*} p<0.05 ;{ }^{* *} p<0.01 ;{ }^{* * *} p<0.001$

Table 5. Mixed-effects model for the effects of left occipital boundary controllability, session, and TMS in the open task

\begin{tabular}{lrrrc}
\hline & Estimate & \multicolumn{1}{l}{ SE } & \multicolumn{1}{l}{$t$-value } & $p$ \\
\hline Intercept & 7.646 & 0.200 & 38.192 & $<0.001^{* * *}$ \\
Session & -1.651 & 0.800 & -2.063 & $0.039^{*}$ \\
ActiveSham & -0.392 & 0.257 & -1.524 & 0.138 \\
Boundary & -0.006 & 0.002 & -2.382 & $0.023^{*}$ \\
Session*Boundary & 0.002 & 0.001 & 2.073 & $0.038^{*}$ \\
Session*ActiveSham & 0.244 & 0.102 & 2.387 & $0.017^{*}$ \\
Boundary*ActiveSham & 0.005 & 0.003 & 1.737 & 0.093 \\
Boundary*ActiveSham*Session & -0.004 & 0.001 & -3.037 & $0.002^{* *}$ \\
\hline
\end{tabular}

ActiveSham, Active TMS effect relative to sham; boundary, continuous boundary controllability effect; session, effect of the second relative to the first session.

${ }^{*} p<0.05 ;{ }^{* *} p<0.01 ;{ }^{* * *} p<0.001$.

ularly important for retrieving words when several alternative words compete for selection, consistent with accounts of the IFG mediating retrieval and selection of distributed representations in semantic memory (Bedny et al., 2008, Pulvermüller, 2013). Speculatively, if the LIFG is relatively better positioned to control high-level intermodular communication, then increased processing demands may confer more interference with sustained task performance. Therefore, inhibition of the LIFG may release accumulating competition among valid responses that results in practice-related interference for open-ended tasks. This speculation is tenable given that the theoretical role of stronger boundary controllers is to facilitate interactions among major brain networks, which could simultaneously confer advantages to general integrated processing demands but disadvantages as competition between semantically activated representations accumulates.

In contrast, modal controllability within the LIFG was associated with TMS effects in the task with closed-ended language demands, indicating that modal controllability mediates controlled language processes that require specific responses. This observation suggests that the LIFG's theoretical ability to drive the network into difficult-to-reach states (Pasqualetti et al., 2014, Gu et al., 2015) could partially mediate the retrieval and selection of task appropriate responses according to task demands, consistent with some network-level accounts for these processes (Friederici and Singer, 2015, Ye et al., 2014, Fedorenko and Thompson-Schill, 2014). The observed enhanced practice effect after TMS in individuals with low modal controllability may occur due to a suppression of the LIFG's role in multiple local processing demands (Nicolo et al., 2016, Vuksanović et al., 2015) due to an increased number of connections within the network (i.e., lower modal controllability associated with denser connections).

We additionally found that boundary controllability, and not modal controllability, in the left lateral occipital cortex was related to TMS effects on performance in the open-ended (and not closed-ended) tasks. This could be because boundary controllability theoretically describes the roles of regions in guiding largescale integration and segregation of network-level processes in the brain. In the pathway from the left lateral occipital cortex to the left IFG (and current site of stimulation), both regions could variably guide large-scale hierarchical processing demands and TMS influences could be distributed across the network. A future study could test this by directly stimulating the LIFG and lateral occipital cortex with functional neuroimaging. In contrast, on a task in which subjects must retrieve a specific exemplar, the process is more localized to IFG as a function of that region's theoretical role in driving the brain into hard-to-reach states. This dissociation could illustrate a general and important role of highlevel network integration and segregation across regions involved in visually presented language tasks with open-ended response demands. In addition, it highlights the specificity of modal controllability's role in selecting specific responses.

Collectively, these findings provide a basis for integrating the local influences of TMS, mediating anatomical organization across the brain and performance on controlled language tasks. Speculatively, our results could promote work that aims to connect neurophysiological and network neuroscience (Medaglia et al., 2015, Bassett and Sporns, 2017) more generally. Although the LIFG may be related to control functions generally (Brass et al., 2005), it may be possible to pair TMS, diffusion imaging, and task manipulations to dissociate specific contributions outside the language domains. In addition, the executive processes involved in language may not be unique to the frontal lobe (Whitney et al., 2011) and whether similar network controllers in other parts of 
the brain influence controlled language function remains to be seen.

It is important to note that, presumably, GABA-mediated inhibition (Trippe et al., 2009) is a consequence of the direct effects of repetitive TMS and a potential mediating mechanism for variability in word selection processes (Snyder et al., 2010). However, this local mechanism is situated in the context of individually variable anatomical networks, which our current work establishes are relevant to dissociable control processes in the brain associated with variability in language task performance. Some cross-sectional work illustrates the link between anatomical network controllability and fMRI dynamics across neurodevelopment (Tang et al., 2017). Although the current study used a simplified model of dynamics that has been demonstrated to predict the controllability of Wilson-Cowan (Muldoon et al., 2016) and Kuramoto (Tiberi et al., 2017) oscillators coupled with empirically measured anatomical brain networks, it is important to note that theoretical predictions about controllability would be further strengthened by evaluating empirically measured neural activity in response to exogenous brain stimulation. For example, demonstrating that integrated or segregated or difficult to reach BOLD or EEG states are influenced by TMS as a function of boundary and modal controllability, respectively, would support the theoretical notions described here. Validating dynamic predictions with concurrent or post-TMS data will be crucial to understand the dynamic shifts responsible for behavior change.

Future studies could examine larger cohorts, including the effects of TMS at the LIFG over broader age ranges and in patients with neuropsychiatric conditions. Although the multilevel modeling approach used here implicitly accounts for biased estimates associated with uneven sample sizes and variances in the betweensubjects condition examined, it may still come at the cost of a slight reduction in power (Bell et al., 2014). Future studies should investigate the relationship between TMS effects with larger and evenly sized samples to replicate and extend current findings, potentially revealing more subtle effects resulting from TMS. In addition, whereas the current results establish a link between TMS boundary controllability and response times during controlled language function, they are not specific to classically examined selection or retrieval demands at the item level. We could thus examine the interactions between the LIFG and specific controllable subnetworks of the brain involved in more general or specific control processes (Fedorenko and Thompson-Schill, 2014) and different behavioral task designs such as open-ended number generation and closed-ended sentence paradigms to examine relationships between network controllability and varying retrieval and selection response demands.

Here, we applied a TBS sequence, but numerous other stimulation procedures have been used to influence cognitive-emotional functioning, including in the LIFG. Future studies could use varying stimulation parameters to examine the sensitivity of controlled language function to different stimulation intensities, as well as their interaction with network controllability. In addition, whereas our network statistics were related to behavior, our sham procedure does not rule out the possibility that muscle contraction related effects at the IFG are related to performance variability in the TMS condition. We also selected a vertex sham stimulation to limit identifiability of the sham coil orientation and to simplify the interpretation of network-mediated effects to one site. Importantly, in some cases, active stimulation had opposing effects on response latencies depending on the LIFG's network control role and task demands, arguing against a general effect of stimulation. However, future research should explore sham stimulation at the IFG or active stimulation at a control site to extend the current findings. Finally, although we used methods consistent with earlier work applying controllability analysis to anatomical networks (Gu et al., 2015, Betzel et al., 2016, Tang et al., 2017, Gu et al., 2017), future studies could investigate the optimal parameters for predicting TMS effects including selection of fiber tracking, parcellation, and network weighting or binarizing procedures.

By examining the relationship between interindividual variability in LIFG controllability and controlled language function before and after brain stimulation, we have established a bridge between a neuroscientist's notion of a controlled language process and an engineer's notion of network control. The current results demonstrate that linking network controllability in white matter networks with experimental manipulation involving TMS can reveal associations between regional network control roles and cognitive susceptibility to brain stimulation. It is possible to identify dissociable control roles of a single region of the brain using simplified dynamic models in anatomical connectivity. Similar experiments may elucidate the role of the LIFG in specific and general cognitive control functions in the human connectome.

\section{References}

Abdelnour F, Voss HU, Raj A (2014) Network diffusion accurately models the relationship between structural and functional brain connectivity networks. Neuroimage 90:335-347. CrossRef Medline

Baayen RH (2008) Analyzing linguistic data: A practical introduction to statistics using R. Cambridge, UK: Cambridge University.

Baayen RH, Davidson DJ, Bates DM (2008) Mixed-effects modeling with crossed random effects for subjects and items. J Mem Lang 59:390-412. CrossRef

Barr DJ, Levy R, Scheepers C, Tily HJ (2013) Random effects structure for confirmatory hypothesis testing: keep it maximal. J Mem Lang 68:255278. CrossRef

Bassett DS, Sporns O (2017) Network neuroscience. Nat Neurosci 20:353364. CrossRef Medline

Bassett DS, Brown JA, Deshpande V, Carlson JM, Grafton ST (2011) Conserved and variable architecture of human white matter connectivity. Neuroimage 54:1262-1279. CrossRef Medline

Bates D, Maechler M, Bolker B, Walker S (2015). Fitting Linear Mixed-Effects Models Using lme4. J Stat Software 67:1-48. CrossRef

Bedny M, McGill M, Thompson-Schill SL (2008) Semantic adaptation and competition during word comprehension. Cereb Cortex 18:2574-2585. CrossRef Medline

Bell BA, Morgan GB, Schoeneberger JA, Kromrey JD, Ferron JM (2014) How low can you go? An investigation of the influence of sample size and model complexity on point and interval estimates in two-level linear models. Methodology: European Journal of Research Methods for the Behavioral and Social Sciences 10:1. CrossRef

Benali A, Trippe J, Weiler E, Mix A, Petrasch-Parwez E, Girzalsky W, Eysel UT, Erdmann R, Funke K (2011) Theta-burst transcranial magnetic stimulation alters cortical inhibition. J Neurosci 31:1193-1203. CrossRef Medline

Benedek M, Beaty R, Jauk E, Koschutnig K, Fink A, Silvia PJ, Dunst B, Neubauer AC (2014) Creating metaphors: the neural basis of figurative language production. Neuroimage 90:99-106. CrossRef Medline

Betzel RF, Gu S, Medaglia JD, Pasqualetti F, Bassett DS (2016) Optimally controlling the human connectome: the role of network topology. Sci Rep 6.

Blondel VD, Guillaume JL, Lambiotte R, Lefebvre E (2008) Fast unfolding of communities in large networks. Journal of Statistical Mechanics: Theory and Experiment 10:P1000.

Botvinick MM, Braver TS, Barch DM, Carter CS, Cohen JD (2001) Conflict monitoring and cognitive control. Psychol Rev 108:624-652. CrossRef Medline

Brass M, Derrfuss J, Forstmann B, von Cramon DY (2005) The role of the inferior frontal junction area in cognitive control. Trends Cogn Sci 9:314-316. CrossRef Medline

Cai Q, Van der Haegen L, Brysbaert M (2013) Complementary hemispheric 
specialization for language production and visuospatial attention. Proc Natl Acad Sci U S A 110:E322-E330. CrossRef Medline

Cammoun L, Gigandet X, Meskaldji D, Thiran JP, Sporns O, Do KQ, Maeder P, Meuli R, Hagmann P (2012) Mapping the human connectome at multiple scales with diffusion spectrum MRI. J Neurosci Methods 203: 386-397. CrossRef Medline

Chai LR, Mattar MG, Blank IA, Fedorenko E, Bassett DS (2016) Functional network dynamics of the language system. Cereb Cortex 26:4148-4159. CrossRef Medline

Cieslak M, Grafton ST (2014) Local termination pattern analysis: a tool for comparing white matter morphology. Brain Imaging Behav 8:292-299. CrossRef Medline

Doron KW, Bassett DS, Gazzaniga MS (2012) Dynamic network structure of interhemispheric coordination. Proc Natl Acad Sci U S A 109:1866118668. CrossRef Medline

Fedorenko E, Thompson-Schill SL (2014) Reworking the language network. Trends Cogn Sci 18:120-126. CrossRef Medline

Fischl B (2012) Freesurfer. Neuroimage 62:774-781. CrossRef Medline

Friederici AD, Singer W (2015) Grounding language processing on basic neurophysiological principles. Trends Cogn Sci 19:329-338. CrossRef Medline

Gernsbacher MA, Kaschak MP (2003) Neuroimaging studies of language production and comprehension. Annu Rev Psychol 54:91-114. CrossRef Medline

Good BH, de Montjoye YA, Clauset A (2010) Performance of modularity maximization in practical contexts. Phys Rev E Stat Nonlin Soft Matter Phys 81:046106. CrossRef Medline

Gu S, Pasqualetti F, Cieslak M, Telesford QK, Yu AB, Kahn AE, Medaglia JD, Vettel JM, Miller MB, Grafton ST, Bassett DS (2015) Controllability of structural brain networks. Nat Commun 6:8414. CrossRef Medline

Gu S, Betzel RF, Mattar MG, Cieslak M, Delio PR, Grafton ST, Pasqualetti F, Bassett DS (2017) Optimal trajectories of brain state transitions. Neuroimage 148:305-317. CrossRef Medline

Hagmann P, Cammoun L, Gigandet X, Meuli R, Honey CJ, Wedeen VJ, Sporns O (2008) Mapping the structural core of human cerebral cortex. PLoS Biol 6:e159. CrossRef Medline

Hamdan AMA, Nayfeh AH (1989) Measures of modal controllability and observability for first-and second-order linear systems. AIAA Journal of Guidance, Control, and Dynamics 12:421-428. CrossRef

Hermundstad AM, Bassett DS, Brown KS, Aminoff EM, Clewett D, Freeman S, Frithsen A, Johnson A, Tipper CM, Miller MB, Grafton ST, Carlson JM (2013) Structural foundations of resting-state and task-based functional connectivity in the human brain. Proc Natl Acad Sci U S A 110:61696174. CrossRef Medline

Hermundstad AM, Brown KS, Bassett DS, Aminoff EM, Frithsen A, Johnson A, Tipper CM, Miller MB, Grafton ST, Carlson JM (2014) Structurallyconstrained relationships between cognitive states in the human brain. PLoS Comput Biol 10:e1003591. CrossRef Medline

Hoffman P, Jefferies E, Lambon Ralph MA (2010) Ventrolateral prefrontal cortex plays an executive regulation role in comprehension of abstract words: convergent neuropsychological and repetitive tms evidence. J Neurosci 30:15450-15456. CrossRef Medline

Hoffman P, Lambon Ralph MA, Rogers TT (2013) Semantic diversity: a measure of semantic ambiguity based on variability in the contextual usage of words. Behav Res Methods 45:718-730. CrossRef Medline

Honey CJ, Thivierge JP, Sporns O (2010) Can structure predict function in the human brain? Neuroimage 52:766-776. CrossRef Medline

Honey CJ, Sporns O, Cammoun L, Gigandet X, Thiran JP, Meuli R, Hagmann P (2009) Predicting human resting-state functional connectivity from structural connectivity. Proc Natl Acad Sci U S A 106:2035-2040. CrossRef Medline

Huang YZ, Edwards MJ, Rounis E, Bhatia KP, Rothwell JC (2005) Theta burst stimulation of the human motor cortex. Neuron 45:201-206. CrossRef Medline

Jefferies E (2013) The neural basis of semantic cognition: converging evidence from neuropsychology, neuroimaging and TMS. Cortex 49:611625. CrossRef Medline

Jefferies E, Lambon Ralph MA (2006) Semantic impairment in stroke aphasia versus semantic dementia: a case-series comparison. Brain 129:21322147. CrossRef Medline

Jutla IS, Jeub LGS, Mucha PJ (2011) A generalized Louvain method for community detection implemented in MATLAB. Available at http://netwiki.amath.unc.edu/GenLouvain.

Kailath T (1980) Linear systems. Upper Saddle River, NJ: Prentice-Hall.

Kalman RE, Ho YC, Narendra SK (1963) Controllability of linear dynamical systems. Contributions to Differential Equations 1:189-213.

Krieger-Redwood K, Jefferies E (2014) TMS interferes with lexical-semantic retrieval in left inferior frontal gyrus and posterior middle temporal gyrus: Evidence from cyclical picture naming. Neuropsychol 64:24-32. CrossRef Medline

Liu YY, Slotine JJ, Barabási AL (2011) Controllability of complex networks. Nature 473:167-173. CrossRef Medline

Lorch RF Jr, Myers JL (1990) Regression analyses of repeated measures data in cognitive research. J Exp Psychol Learn Mem Cogn 16:149-157. CrossRef Medline

Medaglia JD, Lynall ME, Bassett DS (2015) Cognitive network neuroscience. J Cogn Neurosci 27:1471-1491. CrossRef Medline

Moss HE, Abdallah S, Fletcher P, Bright P, Pilgrim L, Acres K, Tyler LK (2005) Selecting among competing alternatives: selection and retrieval in the left inferior frontal gyrus. Cereb Cortex 15:1723-1735. CrossRef Medline

Muldoon SF, Pasqualetti F, Gu S, Cieslak M, Grafton ST, Vettel JM, Bassett DS (2016) Stimulation-based control of dynamic brain networks. PLoS Comput Biol 12:e1005076. CrossRef Medline

Nelson JK, Reuter-Lorenz PA, Persson J, Sylvester CY, Jonides J (2009) Mapping interference resolution across task domains: a shared control process in left inferior frontal gyrus. Brain Res 1256:92-100. CrossRef Medline

Newman ME (2006) Modularity and community structure in networks. Proc Natl Acad Sci U S A 103:8577-8582. CrossRef Medline

Nicolo P, Fargier R, Laganaro M, Guggisberg AG (2016) Neurobiological correlates of inhibition of the right broca homolog during new-word learning. Front Hum Neurosci 10:371. CrossRef Medline

Noonan KA, Jefferies E, Visser M, Lambon Ralph MA (2013) Going beyond inferior prefrontal involvement in semantic control: evidence for the additional contribution of dorsal angular gyrus and posterior middle temporal cortex. J Cogn Neurosci 25:1824-1850. CrossRef Medline

Pasqualetti F, Zampieri S, Bullo F (2014) Controllability metrics, limitations and algorithms for complex networks. IEEE Transactions on Control of Network Systems 1:40-52. CrossRef

Pulvermüller F (2013) How neurons make meaning: brain mechanisms for embodied and abstract-symbolic semantics. Trends Cogn Sci 17:458470. CrossRef Medline

R Core Team (2016) R: a language and environment for statistical computing. Vienna: R Foundation for Statistical Computing.

Rubinov M, Sporns O (2010) Complex network measures of brain connectivity: uses and interpretations. Neuroimage 52:1059-1069. CrossRef Medline

Ruths J, Ruths D (2014) Control profiles of complex networks. Science 343: 1373-1376. CrossRef Medline

Saur D, Kreher BW, Schnell S, Kümmerer D, Kellmeyer P, Vry MS, Umarova R, Musso M, Glauche V, Abel S, Huber W, Rijntjes M, Hennig J, Weiller C (2008) Ventral and dorsal pathways for language. Proc Natl Acad Sci U S A 105:18035-18040. CrossRef Medline

Schiff SJ (2012) Neural control engineering. Cambridge, MA: MIT.

Segaert K, Menenti L, Weber K, Petersson KM, Hagoort P (2012) Shared syntax in language production and language comprehension? An fMRI study. Cereb Cortex 22:1662-1670. Medline

Snyder HR, Munakata Y (2008) So many options, so little time: The roles of association and competition in underdetermined responding. Psychon Bull Rev 15:1083-1088. CrossRef Medline

Snyder HR, Hutchison N, Nyhus E, Curran T, Banich MT, O'Reilly RC, Munakata Y (2010) Neural inhibition enables selection during language processing. Proc Natl Acad Sci U S A 107:16483-16488. CrossRef Medline

Snyder HR, Banich MT, Munakata Y (2011) Choosing our words: retrieval and selection processes recruit shared neural substrates in left ventrolateral prefrontal cortex. J Cogn Neurosci 23:3470-3482. CrossRef Medline

Snyder HR, Kaiser RH, Whisman MA, Turner AE, Guild RM, Munakata Y (2014) Opposite effects of anxiety and depressive symptoms on executive function: the case of selecting among competing options. Cogn Emot 28:893-902. CrossRef Medline

Tang E, Giusti C, Baum GL, Gu S, Pollock E, Kahn AE, Roalf DR, Moore TM, Ruparel K, Gur RC, Gur RE, Satterthwaite TD, Bassett DS (2017) Devel- 
opmental increases in white matter network controllability support a growing diversity of brain dynamics. Nat Commun 8:1252. CrossRef Medline

Thompson-Schill SL, Botvinick MM (2006) Resolving conflict: A response to Martin and Cheng (2006). Psychon Bull Rev 13:402-408; discussion 409-411. CrossRef Medline

Thompson-Schill SL, D’Esposito M, Aguirre GK, Farah MJ (1997) Role of left inferior prefrontal cortex in retrieval of semantic knowledge: a reevaluation. Proc Natl Acad Sci U S A 94:14792-14797. CrossRef Medline

Thompson-Schill SL, D’Esposito M, Kan IP (1999) Effects of repetition and competition on activity in left prefrontal cortex during word generation. Neuron 23:513-522. CrossRef Medline

Tiberi L, Favaretto C, Innocenti M, Bassett DS, Pasqualetti F (2017) Synchronization patterns in networks of Kuramoto oscillators: a geometric approach for analysis and control. arXiv preprint arXiv:1709.06193. Available at https://arxiv.org/abs/1709.06193.

Tippett LJ, Gendall A, Farah MJ, Thompson-Schill SL (2004) Selection ability in Alzheimer's disease: investigation of a component of semantic processing. Neuropsychology 18:163-173. CrossRef Medline

Trippe J, Mix A, Aydin-Abidin S, Funke K, Benali A (2009) Theta burst and conventional low-frequency rtms differentially affect GABAergic neurotransmission in the rat cortex. Exp Brain Res 199:411-421. CrossRef Medline

Vuksanović J, Jelić MB, Milanović SD, Kačar K, Konstantinović L, Filipović SR (2015) Improvement of language functions in a chronic non-fluent post-stroke aphasic patient following bilateral sequential theta burst magnetic stimulation. Neurocase 21:244-250. CrossRef Medline

Whitney C, Kirk M, O'Sullivan J, Lambon Ralph MA, Jefferies E (2011) The neural organization of semantic control: tms evidence for a distributed network in left inferior frontal and posterior middle temporal gyrus. Cereb Cortex 21:1066-1075. Medline

Ye Z, Zhou X (2009) Executive control in language processing. Neurosci Biobehav Rev 33:1168-1177. CrossRef Medline

Ye Z, Doñamayor N, Münte TF (2014) Brain network of semantic integration in sentence reading: insights from independent component analysis and graph theoretical analysis. Hum Brain Mapp 35:367-376. CrossRef Medline

Yeh FC, Wedeen VJ, Tseng WY (2011) Estimation of fiber orientation and spin density distribution by diffusion deconvolution. Neuroimage 55 : 1054-1062. CrossRef Medline 